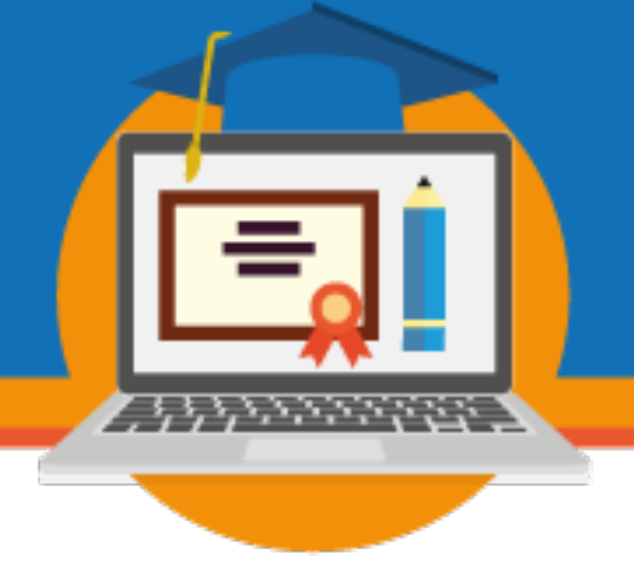

\title{
PLUGIN BLOCO GAME - APLICANDO GAMIFICAÇÃO EM AMBIENTES DE APRENDIZAGEM MOODLE
}

\author{
José Wilson da Costa \\ Secretaria de Educação do Distrito Federal \\ j.wilson.df@gmail.com
}

Eixo 03: Tecnologias, mídias e processos inovadores na EaD

\begin{abstract}
Resumo: O uso de técnicas de gamificação na educação online é uma crescente, essa apresentação visa explorar algumas dessas técnicas e apresentar o plugin Bloco Game como estratégia de gamificação no ambiente virtual de aprendizagem Moodle. Esse plugin constituise em um bloco desenvolvido para o Moodle, que possibilita a aplicação diversas técnicas de gamificação no AVA. O plugin foi desenvolvido utilizando técnicas de gamificação, a saber: Accomplishment (Desenvolvimento e realização), Meaning (Significado), Empowerment (Capacitação da criatividade e feedback), Ownership (Propriedade) e Scarcity (Escassez). O resultado do uso do plugin foi o de que o engajamento dos alunos nos cursos que estão utilizando o bloco é bem superior aos dos alunos nos cursos que não estão utilizando, tanto em participação das atividades propostas, quanto a de conclusão dos cursos.
\end{abstract}

Palavras-chave: Gamificação; Educação online; Plugin; Bloco Game.

\section{Introdução}

Aprender brincando ou brincando de aprender, a gamificação pode ser uma poderosa ferramenta de interação e motivação, capaz de gerar um ganho significativo para os alunos no processo de ensino e aprendizagem (PIMENTA, 2014). Os jogos e atividades colaborativas com suporte computacional podem auxiliar no desenvolvimento de habilidades psicológicas, cognitivas, físicas e sociais (BIASUTTI, 2011; SUNG; HWANG, 2013; ZEA, et al., 2009). No entanto, precisam ser projetados de maneira adequada para que os resultados sejam positivos para os usuários, uma vez que mal projetados podem desencadear baixo nível de aprendizagem e comportamentos de auto-alienação por parte dos alunos/jogadores (SUNG; HWANG, 2013). 


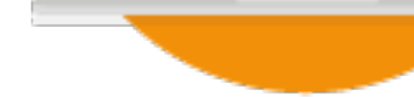

Neste sentido, o desenho de jogos colaborativos com suporte computacional deve estar pautado nos pressupostos da aprendizagem colaborativa online, que pode ser definida como a aprendizagem que enfatiza o grupo e as tarefas colaborativas entre professores e alunos e alunos e alunos, sendo possível evidenciar a interação e a participação no trabalho online (TELES, 2012). A aprendizagem colaborativa online, parte da premissa da construção coletiva do conhecimento, tese que ganha força na educação a distância a medida em que os alunos reconstroem coletivamente os conhecimentos produzidos pela humanidade, tornando-se sujeitos históricos dessa construção coletiva (TEIXEIRA, 2016).

Nesta perspectiva, este artigo discute a gamificação na educação online e apresenta o plugin Bloco Game como estratégia de gamificação no ambiente virtual de aprendizagem Moodle. Esse plugin constitui-se em um bloco desenvolvido para o Moodle, que possibilita aplicar diversas técnicas de gamificação no AVA.

\section{GAMIFICAÇÃO E EDUCAÇÃO ONLINE}

A aprendizagem colaborativa com suporte computacional (Computer-supported collaborative learning - CSCL) é um campo emergente e complexo em suas interações, que estuda como as pessoas podem aprender em grupo com o auxílio do computador. A CSCL considera todos os níveis educacionais formais e não-formais. Salienta-se que a capacidade de combinar aprendizagem colaborativa e suporte computacional é um desafio a ser abordado pela CSCL (STAHL; KOSCHMANN; SUTHERS, 2006). Neste contexto, essa combinação a fim de enriquecer o aprendizado possibilita a inclusão de várias ferramentas e recursos disponíveis ou elaborados para os ambientes virtuais de aprendizagem - AVA's. Esses ambientes são considerados espaços férteis para a mediação pedagógica de um processo educativo colaborativo pautado por iniciativas "gamificadas" (PIMENTA, 2014).

A gamificação é definida por diferentes autores, alguns propõem um conceito mais amplo como é o caso de Deterding, Sicart, Nacke, O'Hara e Dixon (2011), em que a gamificação consiste no uso de elementos de jogos em contextos de não-jogos, até um conceito mais específico, proposto por Pimenta (2014), que define gamificação como um conjunto de

\section{SEMINÁRIO DE EDUCAÇÃO A DISTÂNCIA}

Diálogos sobre EaD e uso das TDIC na educação: regulamentação em tempos recentes 
princípios aplicáveis a qualquer ramo de atividade, uma vez que aproveita a motivação, metas alcançáveis, o desejo das pessoas em realizar objetivos claros e bem definidos, feedback positivo, bem como se utiliza da competição para o atingimento de um comportamento pretendido.

Com base nos conceitos apresentados vale ressaltar a categorização proposta por Werbach e Hunter (2012), que apresentam a modelação da gamificação em três categorias, com base em seus elementos, são elas: dinâmicas, mecanismos e componentes. As dinâmicas estão no topo da pirâmide e elencam restrições, emoções, narrativas, progresso e relacionamento; a seguir tem-se os mecanismos que são compostos por desafios, chances, cooperação e competição, feedback, aquisição de recursos, recompensas, transações e tempos; e por fim os componentes que consistem em realizações, avatares, coleções, combates, presentes, placas de liderança, níveis, pontos, missões, gráfico social, equipes e produtos virtuais. Observa-se a importância de acrescentar na categoria mecanismos o elemento da colaboração, uma vez que os jogos têm grande potencial para estimular o trabalho colaborativo. Segundo Vianna, et al. (2013) a possibilidade de realizar desafios coletivos, por meio de missões claras e estímulos adequados faz com que potencializem os resultados alcançados por meio do trabalho coletivo.

Ressalta-se ainda que de acordo com Zea, et al. (2009) os jogos enquanto ferramentas educativas possuem inúmeras vantagens, dentre elas destacam-se: êxito escolar, pois os alunos que utilizam jogos melhoram sua capacidade de leitura; habilidades cognitivas, uma vez que os jogos proporcionam ambientes de aprendizagem embasados na descoberta e na criatividade; motivação, pois os jogos supõem um estimulo para as crianças, o que facilita o processo de aprendizagem; atenção e concentração, devido a sua natureza lúdica, os jogos aumentam a atenção dos alunos na resolução de problemas concretos.

\section{GAMIFICANDO O MOODLE: O USO DE PLUGINS E O BLOCO GAME}

O Moodle (Modular Object Oriented Distance LEarning) é um Ambiente Virtual de Aprendizagem(AVA), um programa para computador destinado a auxiliar educadores a criar cursos on-line de qualidade. O Moddle é um software de fonte aberta (Open Source Software),

\section{SEMINÁRIO DE EDUCAÇÃO A DISTÂNCIA}




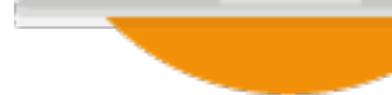

o que significa que se pode instalar, usar, modificar e mesmo distribuir o programa (nos termos da GNU General Public Licence). Pode ser usado, sem modificações, em Unix, Linux, Windows, Mac OS e outros sistemas que suportem PHP. Atualmente, está disponível em mais de 120 idiomas. (MOODLE HQ, 2019)

Por ser de código aberto, o Moodle pode ser personalizado de qualquer forma e adaptado às necessidades individuais. Sua configuração modular e design interoperável permitem que os desenvolvedores criem plugins e integrem aplicativos externos para obter funcionalidades específicas. Pode-se ampliar o que o Moodle faz usando plugins e complementos disponíveis gratuitamente, as possibilidades são infinitas. A maneira mais fácil e sustentável de adicionar novas funcionalidades ao Moodle é instalar um plugin existente que atenda suas necessidades ou desenvolver um plugin com uma nova funcionalidade personalizada para o que precisa.

\subsection{Os plugins e a gamificação}

Os plugins, como o nome diz, servem como "encaixes", funcionam para adicionar recursos aos softwares principais escalonando as funcionalidades e flexibilizando a capacidade de personalização. Com plugins, é preciso apenas "plugar" códigos adicionais nos códigos principais para realizar as mudanças. Caso não haja mais a necessidade dessas mudanças, basta desativar o plugin para “desplugar". Os mesmos são essenciais para aumentar o potencial e as funcionalidades do seu AVA.

É possível encontrar plugins para diversas funcionalidades, no AVA Moodle por exemplo existem 1633 plugins disponíveis em seu diretório e 919 em desenvolvimento, além inúmeros plugins disponíveis em repositórios abertos na internet.

Falando especificamente em gamificação, os próprios AVAs já possuem alguns recursos para aplicar algumas técnicas como por exemplo no Moodle podemos citar os Badges, a barra de progresso para o aluno acompanhar o percentual de conclusão de cada curso, etc. Porém para ampliar o potencial da gamificação no AVA é necessário o uso de plugins.

Um exemplo é o plugin Bloco Game, que possibilita aplicar diversas técnicas de gamificação no AVA Moodle de forma simples e descomplicada. As técnicas citadas a seguir

\section{SEMINÁRIO DE EDUCAÇÃO A DISTÂNCIA}

Diálogos sobre EaD e uso das TDIC na educação: regulamentação em tempos recentes 


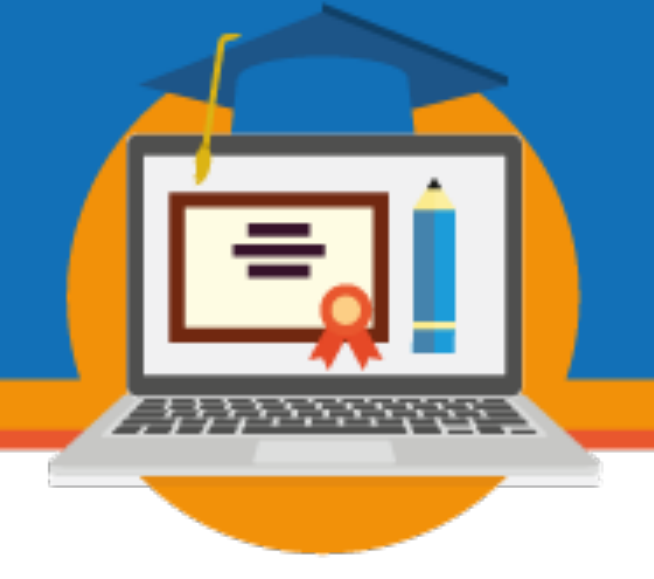

estão embasadas no Framework Octalysis, desenvolvido por Chou (2016). O modelo de Gamificação Octalysis possui 8 perspectivas chamadas de Core Drives (CDs), que se caracterizam como motivadores-chave. Cada um desses Core Drives possui um conjunto de técnicas de gamificação, associadas a eles (CHOU, 2016).

Com base nas técnicas apresentadas para o Octalysis, foram identificadas as que podem ser encontradas no plugin Bloco Games, a seguir apresenta-se a técnica seguida de sua descrição com base na aplicação do plugin Bloco Game.

\section{$\circ \quad$ Accomplishment (Desenvolvimento e realização)}

○ Points: Por meio dessa técnica os professores conseguem aplicar uma pontuação para os estudantes do curso executando atividades, bônus diário e conclusão de curso. Os pontos são utilizados em diversos outros setores como para realizar a classificação, passar de nível, entre outros.

○ Badges: Ao completar um curso o estudante recebe um emblema especial de conclusão, e com este emblema também recebe uma pontuação, a quantidade de emblemas é um fator de desempate para a classificação geral dos estudantes.

○ Leaderboard: Com a pontuação recebida o estudante pode ver sua classificação dentro de um curso especifico, como também de toda a plataforma. O professor ou administrador do moodle pode decidir manter os nomes originais ou ocultar e mostrar somente a posição e quantos pontos os outros teriam.

- LevelUP: O plugin possui um sistema de níveis que evolui conforme o estudante recebe pontos, o avanço de níveis desbloqueia outros recursos na plataforma, como os avatares que este pode utilizar, e a pontuação necessária para avançar cada nível.

○ High Five: Pequenas mensagens de incentivo ao subir de nível.

○ Step-by-Step Tutorial: Tutorial descritivo contendo todas as regras do jogo, o tutorial se adapta dentro da própria plataforma dependendo de quais recursos do plugin o professor optou por utilizar em sua configuração. 

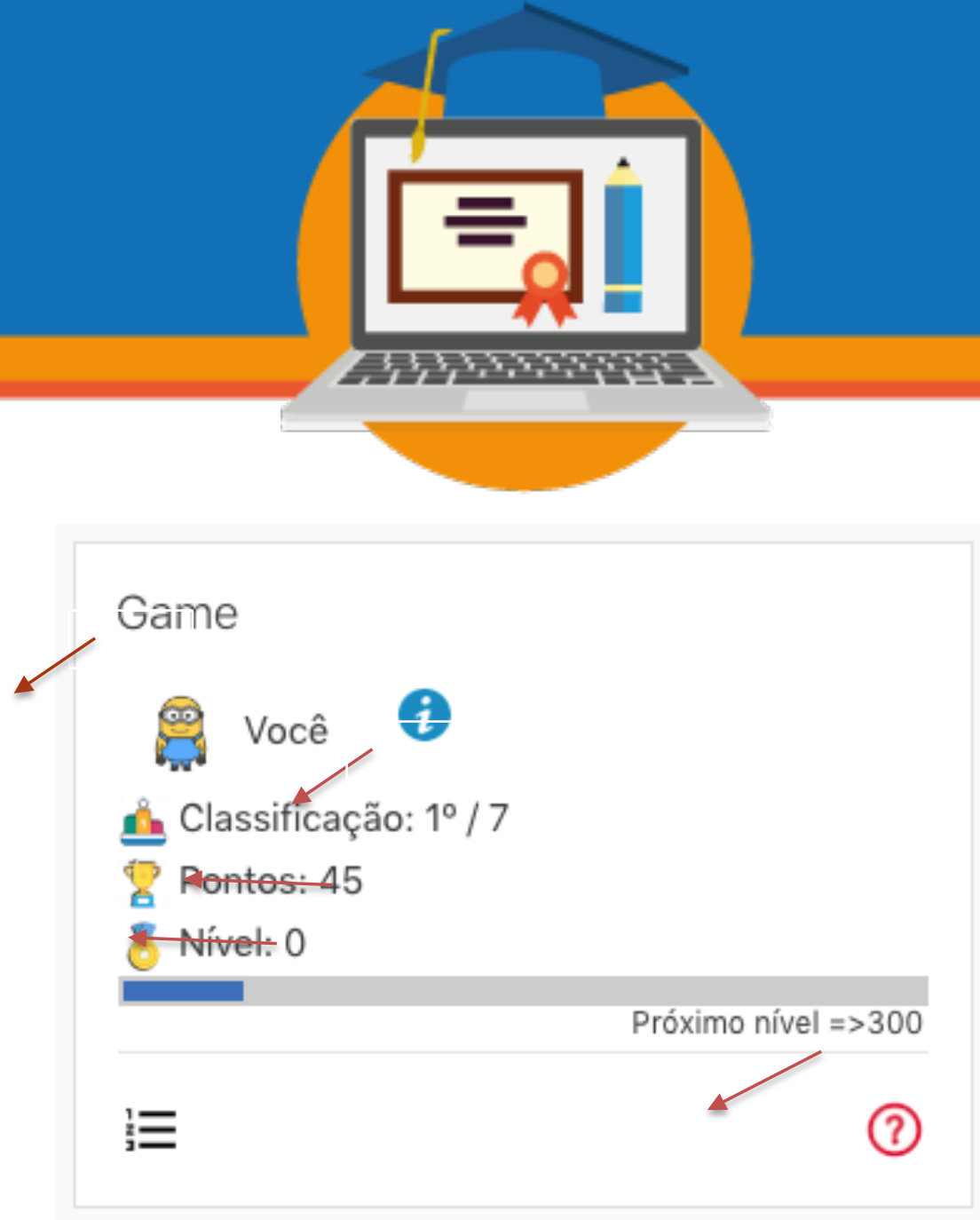

Figura 1 - Bloco do plugin

\section{$\bigcirc \quad$ Meaning (Significado)}

○ Beginners Luck: Os primeiros Avatares são facilmente desbloqueáveis, e conforme o usuário vai passando de nível, o desbloqueio de novos avatares vai se tornando mais raro, necessitando de um maior esforço dos participantes.

○ Free Lunch: Esta técnica é aplicada por meio do recurso de bônus diário, que recompensa o estudante com uma pontuação somente por entrar na plataforma e/ou no curso.

\section{○ Empowerment (Capacitação da criatividade e feedback)}

○ Blank Fills: O professor tem a necessidade de interagir com a plataforma, configurar as técnicas que deseja aplicar.

- General's Carrot: O professor tem uma certa liberdade para configurar o plugin como considerar melhor para sua turma, definindo quais recursos irá utilizar, a quantidade de pontos que os alunos receberão para cada um desses recursos, etc.

○ Instant Feedback: Ao aluno receber uma pontuação, passar de nível ou classificação, o plugin atualiza automaticamente para demonstrar estes novos resultados.

\section{SEMINÁRIO DE EDUCAÇÃO A DISTÂNCIA}




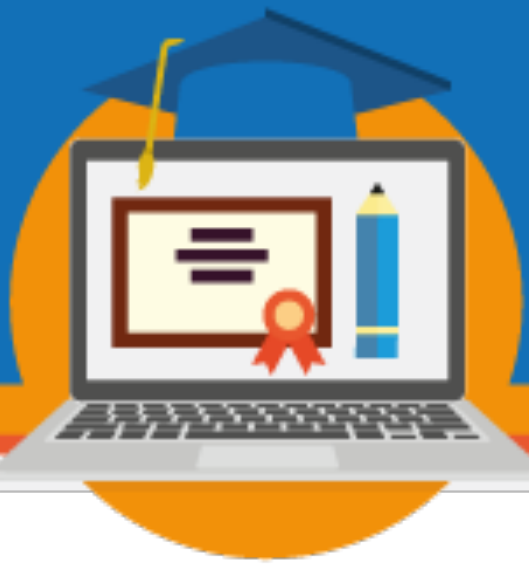

○ Poison Picker: O professor é capaz de ter o controle de recusar a utilização de alguns recursos já oferecidos pelo plugin, como o bônus diário ou classificação por exemplo. Tendo assim um controle dos recursos utilizados em sua turma. 


\section{$E_{1}$

\section{Configurando bloco Game}

\section{- Configurações do bloco}

Alterar título

Mostrar nome do curso

Mostrar informações do jogador

Pontuar notas de atividades

Bônus do dia

Mostrar Classificação

Limite da lista de classificação (0 para ilimitado)

Preservar identidade do usuário

Mostrar Pontos

Mostrar Nível

Número de níveis

Pontos para o nível 1

Pontos para o nível 2

Pontos para o nível 3

Pontos para o nível 4

Pontos para o nível 5

Pontos para o nível 6

Pontos para o nível 7
(2)

?

Não -

$\operatorname{Sim} \hat{-}$

$\operatorname{Sim} \uparrow$

$?$

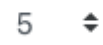

?

$\operatorname{Sim} \leftarrow$

?

$10 \div$

?

$$
\text { Não * }
$$

?

$\operatorname{Sim} 、$

?

$\operatorname{Sim} \leqslant$

?

$12 \div$

?

300

?

$$
500
$$

?

1000

?

$$
2000
$$

?

4000

(2)

6000

? 


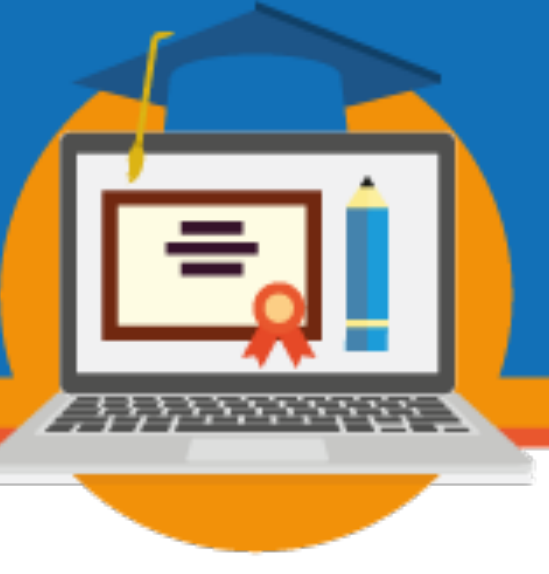

Figura 2 - Configuração do bloco

\section{Ownership (Propriedade)}

○ Virtual Goods: O usuário possui alguns itens que pode receber ao longo do curso, como emblemas, novas imagens de avatares, etc.

○ Avatar: O plugin possui uma série de avatares na qual o usuário poderá escolher como uma representação virtual para sua conta, este avatares podem ser liberados conforme o progresso do estudante no curso, e podem ser modificados na página inicial da plataforma.

\section{Selecione seu Avatar}
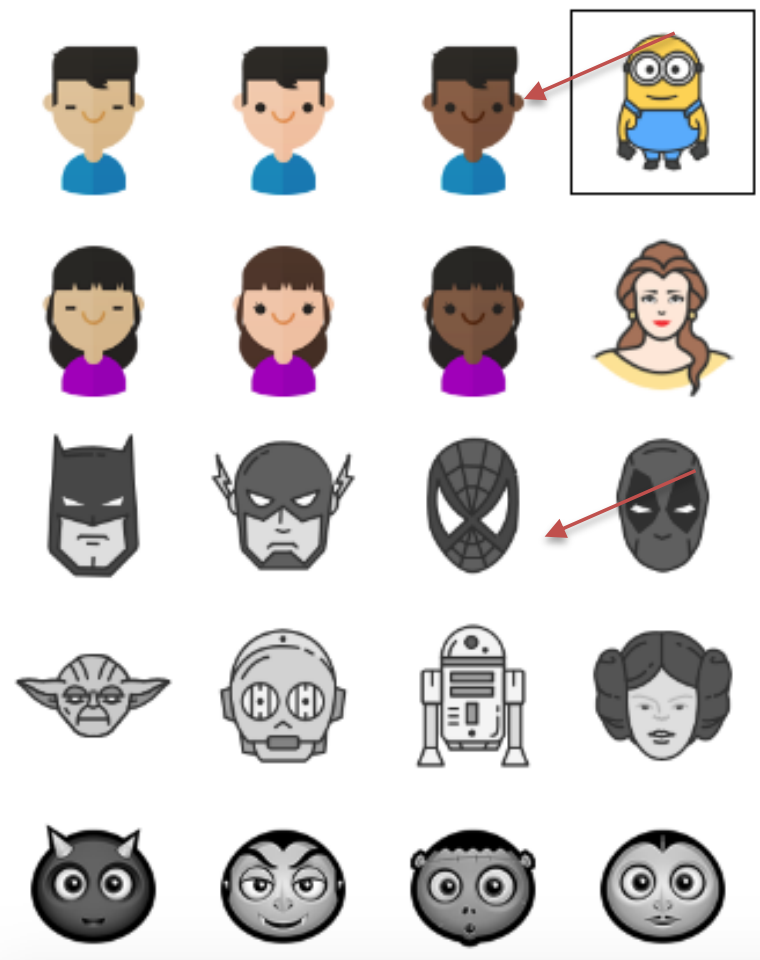

Figura 3 - Página de seleção de Avatar

\section{SEMINÁRIO DE EDUCAÇÃO A DISTÂNCIA}




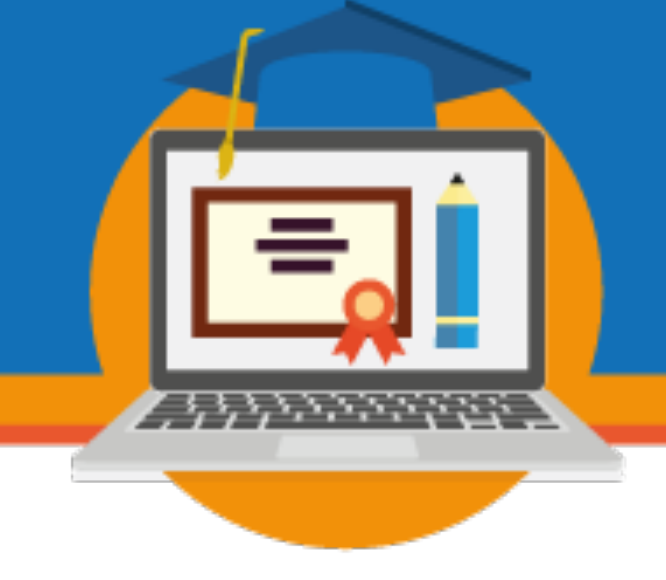

\section{- Scarcity (Escassez)}

○ Dangling: Ao entrar na página para selecionar seu avatar, será mostrado em tons cinzas aqueles que o estudante ainda não desbloqueou e portanto não poderá selecionar, como uma forma de incentivar a continuar estudando e com isto conseguir liberar os restantes.

- Fixed Intervals: O bônus diário ocorre somente na primeira vez que o estudante logar na plataforma no dia, caso este acesse diversas vezes durante o mesmo dia só ganhará os pontos pela primeira, fazendo que ele aguarde até o próximo dia para receber o bônus novamente.

O plugin ainda permite que seja configurado para preservar a identidade dos usuários na listagem de classificação, como mostra a Figura 4.

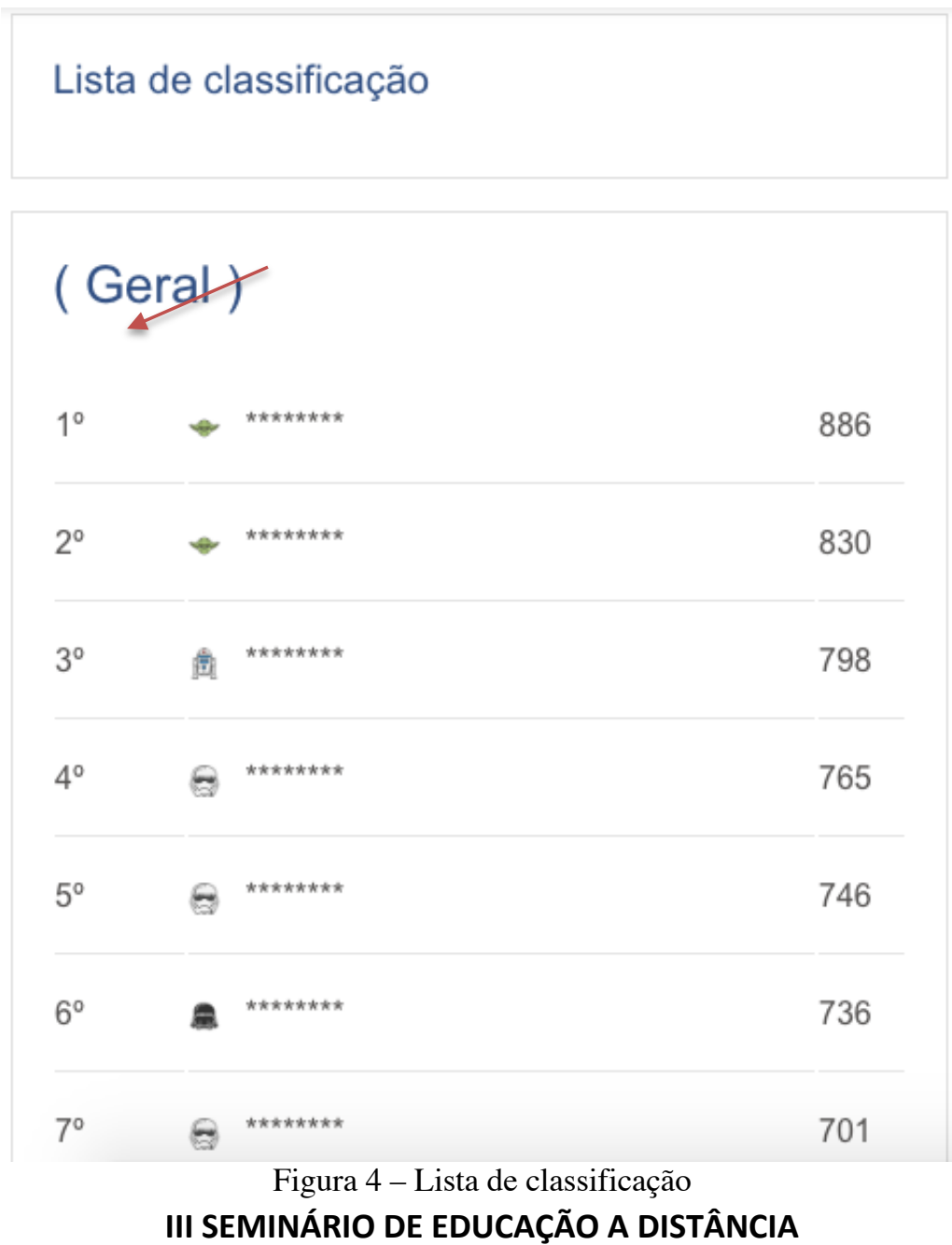

Diálogos sobre EaD e uso das TDIC na educação: regulamentação em tempos recentes 3 a 6 de novembro de 2020 - Brasília/DF - Online 


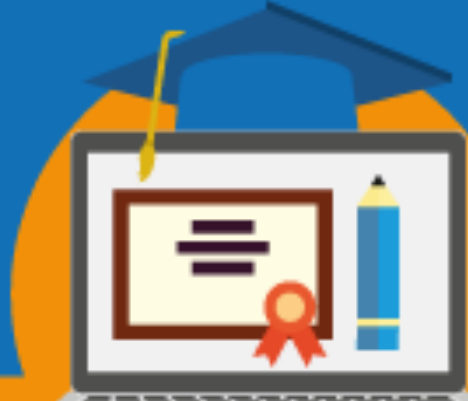

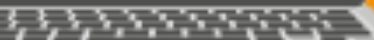

Os testes do plugin foram realizados nas disciplinas de Desenvolvimento de Projetos, Linguagem Técnica de Programação III, Inglês Técnico II e Empreendedorismo do curso Técnico em Informática na Escola Técnica de Brasília, no período de 29/06 a 29/11/2019. No AVA do curso, o Bloco Game foi inserido com o objetivo de tornar a aprendizagem dos alunos mais prazerosa e criativa, e assim reduzir a evasão.

No conjunto de 328 estudantes, o resultado foi o de que os cursos que estão utilizando o Bloco Game (Desenvolvimento de Projetos, Inglês Técnico II, Linguagem Técnica de Programação III e Empreendedorismo) tiveram melhores resultados em termos de conclusão e evasão do que os cursos que não utilizaram o plugin (Matemática Aplicada, Português Instrumental, Linguagem Técnica de Programação I, Linguagem Técnica de Programação II), isso pode ser observado na Figura 5.

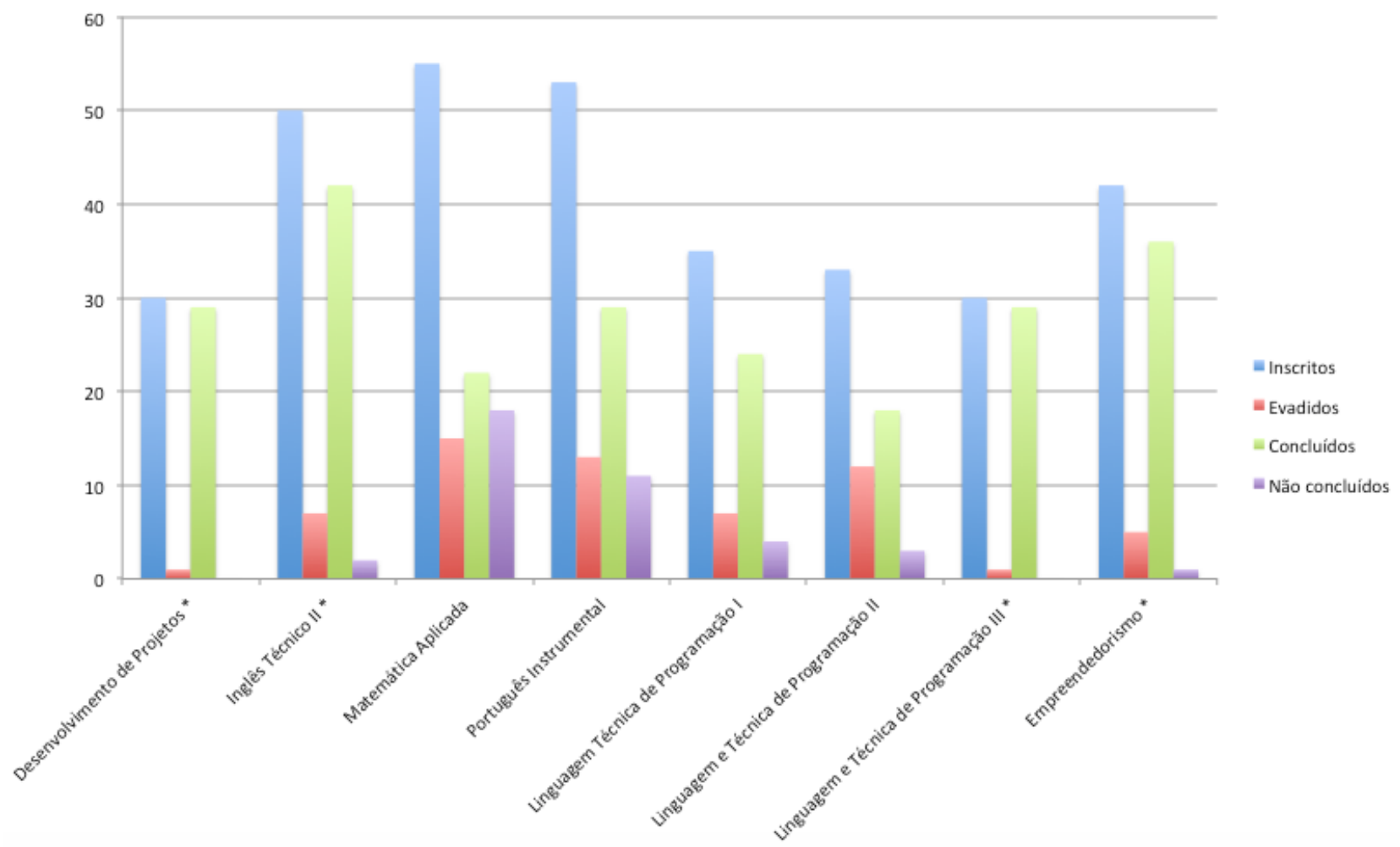

Figura 5 - Análise de dados das disciplinas 


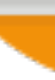

Observa-se ainda que a participação dos alunos nas atividades propostas nos cursos gamificados é superior, o que corrobora a afirmação de Mattar (2014) que os jogos devem ser criativos e ter a capacidade de prender a atenção dos alunos.

Isto posto, foi possível observar por meio dos feedbacks dos alunos o aumento do interesse em relação as atividades gamificadas. A utilização de um ambiente pautado em descobertas e na criatividade proporcionou uma aprendizagem mais prazerosa e criativa o que gerou resultados mais positivos em termos de conclusão e evasão.

\section{CONSIDERAÇÕES FINAIS}

O presente artigo discutiu estratégias de gamificação na educação online por meio do desenvolvimento e implementação do plugin Bloco Game no ambiente virtual de aprendizagem Moodle. Observa-se que existe a necessidade de formação docente para o uso da gamificação em sala de aula, pois essa utilização deve estar atrelada ao planejamento do curso/disciplina.

As atividades propostas devem considerar um ambiente de descobertas embasados na criatividade, com objetivos claros e bem definidos que proporcionem motivação, desafios, chances, cooperação e competição, feedback, aquisição de recursos, recompensas, entre outros (Zea, et al., 2009; Werbach e Hunter, 2012; Pimenta, 2014). Entende-se que o uso sem o devido planejamento e contextualização poderá gerar resultados indesejados, como ressalta Sung e Hwang (2013) uma vez que mal projetados os jogos podem desencadear baixo nível de aprendizagem e comportamentos de auto-alienação por parte dos alunos/jogadores.

A partir da aplicação de técnicas de gamificação de forma planejada e organizada, o uso do Bloco Game demonstrou, nas disciplinas Desenvolvimento de Projetos, Inglês Técnico II, Linguagem Técnica de Programação III e Empreendedorismo, como a utilização de plugins pode potencializar a aprendizagem dos estudantes por meio do AVA.

Como agenda de pesquisa aponta-se a necessidade de estudos que verifiquem a efetividade do Plugin Bloco Game em outros cursos/disciplinas de contextos diferentes ao aplicado, bem como pesquisas que possam auxiliar o professor a escolher quais plugins utilizar e como aplicar os recursos de cada um de forma a explorar todo o seu potencial.

\section{SEMINÁRIO DE EDUCAÇÃO A DISTÂNCIA}

Diálogos sobre EaD e uso das TDIC na educação: regulamentação em tempos recentes 3 a 6 de novembro de 2020 - Brasília/DF - Online 


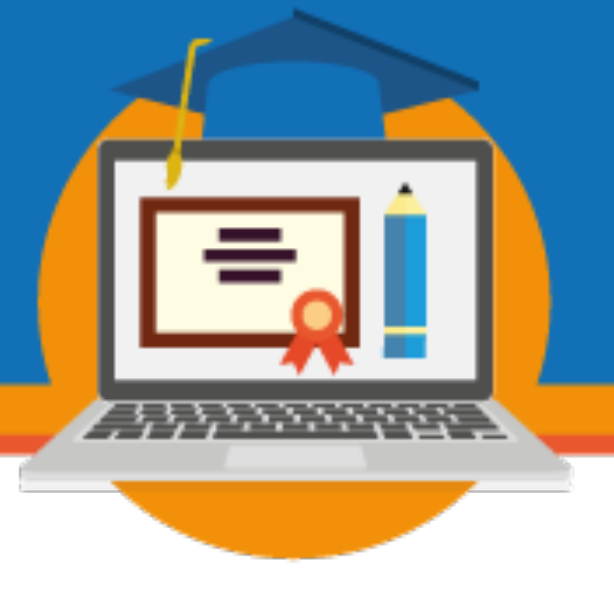

\section{REFERÊNCIAS}

BIASUTTI, Michele. The student experience of a collaborative e-learning university module. Computers \& Education, v. 57, n. 3, p. 1865-1875, 2011.

CHANG, Shujen L. Book Review: The Systematic Design of Instruction (6th Editrion). Walter Dick, Lou Carey; James O. Carey. Educational Technology Research and Development, Vol. 54, nº . 4, p. 417-420, 2009.

CHOU, Y. k. Actionable gamification: beyond points ,badges, and leaderboards. Fremont, CA: Octalysis Media, 2016.

CRIADO, Marta Ortiz; SALGADO, Sonia Medina; DURÁN, Carmen De La Calle. Herramientas para el aprendizaje colaborativo: una aplicación práctica del juego de rol. Teoria de la Educación - Educación y Cultura em la Sociedad de la Información, 2010.

CUPANI, Alberto. La peculiaridad del conocimiento tecnológico. ScientiaeStudia, São Paulo, v. 4, n. 3, p. 353-71, 2006. Disponível em: < http://www .scielo.br/pdf/ss/v4n3/ a01v4n3.pdf>. Acesso em: 29 nov 2019.

DETERDING, S., SICART, M., NACKE, L., O'HARA, K., and DIXON, D. Gamification: Using game-design elements in nongaming contexts. Proc. CHI EA '11, ACM Press (2011), 24252428.

DETERDING, Sebastian et al. From game design elements to gamefulness: defining gamification. In: Proceedings of the 15th international academic MindTrek conference: Envisioning future media environments. ACM, 2011.p. 9-15.

DILLENBOURG, Pierre. What do you mean by collaborative learning. Collaborativelearning: Cognitive and computational approaches, v. 1, p. 1-15, 1999.

FILATRO, Andrea. Design instrucional na prática. São Paulo: Pearson Education do Brasil, 2008.

FREITAS, M. T. A. O ensinar e o aprender na sala de aula... Cadernos para o professor, Juiz de Fora, v. VI, n. 6, p. 6-13, 1998.

FUKS, Hugo et al. Novas Estratégias de Avaliação Online: aplicações e implicações em um curso totalmente a distância através do ambiente AulaNet. Avaliação da Aprendizagem em Educação Online, M. SILVA e E.SANTOS (orgs.), São Paulo: Loyola, p. 369-385, 2006.

GORDON, Jack; ZEMKE, Ron. The attack on ISD. Training, v. 37, n. 4, p. 42-45, 2000.

III SEMINÁRIO DE EDUCAÇÃO A DISTÂNCIA

Diálogos sobre EaD e uso das TDIC na educação: regulamentação em tempos recentes 3 a 6 de novembro de 2020 - Brasília/DF - Online 


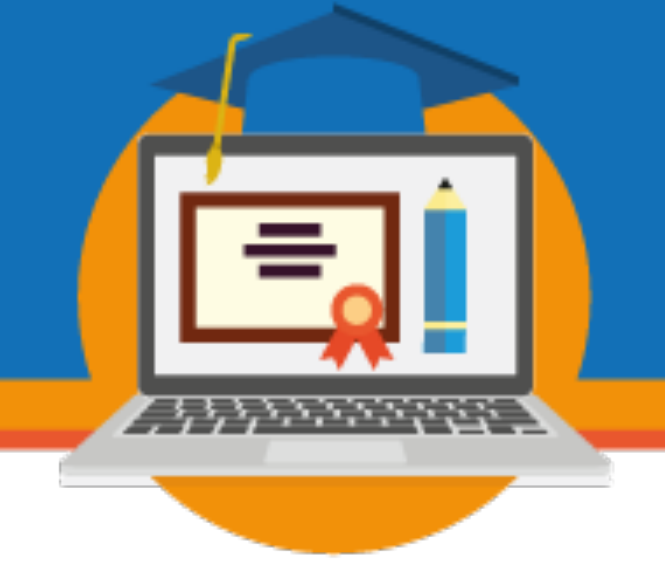

LUCENA, Carlos José Pereira de; FUKS, Hugo; Educação na Era da Internet. Rio de Janeiro: Editora Clube do Futuro, Brasil, 2000.

MATTAR, João. Design educacional: educação a distância na prática. São Paulo: Artesanato Educacional, 2014.

MATTAR, João; NESTERIUK, Sérgio. Estratégias do Design de Games que podem ser incorporadas à Educação a Distância/Game Design Strategies that can be incorporated into Distance Education. Revista Iberoamericana de Educación a Distancia, v. 19, n. 2, p. 91, 2016.

MOODLE HQ, Documentation: About Moodle, 2019. Página Oficial. Disponível em: <https://docs.moodle.org/38/en/About_Moodle>. Acesso em: 29 de novembro de 2019.

PIMENTA, Fabrícia. Faleiros. Gamificação na Aprendizagem Colaborativa Online Não é um jogo, acredite! Não são só pontos, recompensas e desafios...Relatório de pesquisa de Pós Doutorado. Faculdade de Educação da UnB, 2014.

SANTOS, Rosimeire Martins Régis dos. O Processo de colaboração na educação online: interação mediada pelas tecnologias de informação e comunicação. Campo Grande. 2008. 174f. Dissertação (Mestrado em Educação) - Universidade Católica Dom Bosco.

SCHLEMMER, Eliane. Games e Gamificação: uma alternativa aos modelos de EaD. RIED. Revista Iberoamericana de Educación a Distancia, v. 19, n. 2, 2016.

SILVA, Alessandra Lisboa da. Análise da estratégia pedagógica de pequenos grupos colaborativos online de uma disciplina do curso de pedagogia a distância da Universidade de Brasília. Brasília, 2013. Tese (Doutorado) - Programa de Pós-Graduação em Educação da UnB. Disponível em: http://repositorio.unb.br/handle/10482/13476, acesso em: 01/04/2014.

STAHL, Gerry; KOSCHMANN, Timothy; SUTHERS, Dan. Computer-supported collaborative learning: An historical perspective. Cambridge handbook of the learning sciences, v. 2006, p. 409-426, 2006.

SPINK, Mary Jane; MENEGON, Vera Mincoff; MEDRADO, Benedito. Oficinas como estratégia de pesquisa: articulações teórico-metodológicas e aplicações ético-políticas. Revista Psicologia \& Sociedade, v. 26, n. 1, 2014.

SUNG, Han-Yu; HWANG, Gwo-Jen. A collaborative game-based learning approach to improving students' learning performance in science courses. Computers \& Education, v. 63, p. 43-51, 2013. 


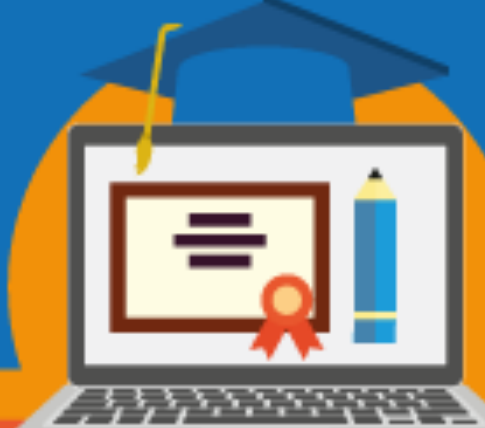

TELES, Lúcio. Aprendizagem colaborativa online. Reflexões e Práticas. $1^{\circ}$ ed: Novas, 2012.

TELES, Lúcio. Dimensões da aprendizagem colaborativa no design e gerenciamento de ambientes online. Artefactum, Rio de Janeiro, v. 13, p. 23-44, 2015.

TEIXEIRA, Janaina Angelina. Formação de Professores-Tutores sob a Perspectiva da Colaboração, Interação e Aprendizagem Significativa na Educação a Distância. Revista Aprendizagem em EAD, v. 5, n. 1, 2016.

VIANNA, Ysmar et al. Gamification, Inc: como reinventar empresas a partir de jogos. Rio de Janeiro: MJV, 2013.

ZEA, Natalia Padilla et al. Diseño de Videojuegos Colaborativos y Educativos Centrado en la Jugabilidad. IEEE-RITA, v. 4, n. 3, p. 191-198, 2009.

WERBACH, Kevin; HUNTER, D. The gamification toolkit. 2012. 